\title{
43629 - EFFECTS OF TERBUTALINE COMBINED WITH GLIBENCLAMIDE ON RATS WITH ENDOTOXIC SHOCK
}

\author{
Wen-Jinn Liaw, Department of Anesthesiology, Tri-Service General Hospital, Taipei, \\ Taiwan, R.O.C.; \\ Nei-Tsu Liu, Institute and Department of Pharmacology; \\ Chin-Chen Wu, Institute and Department of Pharmacology;
}

Background: Our previous studies showed that terbutaline (TB) or glibenclamide (GB) prevents circulatory failure in rats treated with endotoxin (E. coli lipopolyssacharide, LPS). However, the protective effects of these two compounds were only partial and not therapeutic. Thus, this study was further designed to examine the therapeutic effect of $\mathrm{TB}+\mathrm{GB}$ or GB + TB on rats with endotoxic shock.

Methods: Local IRB approval was obtained for this study. Four groups, i.e. sham, LPS (10 $\mathrm{mg} / \mathrm{kg}$, at time 0$), \mathrm{LPS}+\mathrm{GB}(1 \mathrm{mg} / \mathrm{kg}$, at $90 \mathrm{~min})+\mathrm{TB}(0.3 \mathrm{mg} / \mathrm{kg}$, at $3 \mathrm{~h})$ and LPS + TB $(0.3$ $\mathrm{mg} / \mathrm{kg}$, at $30 \mathrm{~min})+\mathrm{GB}(1 \mathrm{mg} / \mathrm{kg}$, at $2 \mathrm{~h})$ were studied. Hemodynamic changes were continuously monitored and animals were sacrificed at the end of in vivo experiments. Thoracic aortas were harvested for the measurement of O2-. Plasma levels of nitrate (an indicator of NO), glutamate pyruvate transaminase (GPT), glutamate oxaloacetate transaminase (GOT), albumin, total protein, creatinine, and calcium as well as blood glucose were also determined. In addition, survival studies were performed in ICR mice treated with a lethal dose of LPS $(60 \mathrm{mg} / \mathrm{kg}$, s.c.) with or without TB + GB or GB + TB until $60 \mathrm{~h}$.

Results: Changes of mean arterial pressure (MAP), heart rate (HR), pressor responses to norepinephrine (NE) and blood glucose were significantly reduced or increased in rats treated with LPS, and the treatment of rats with TB combined with GB fully restored the MAP, but had no significant effect on blood glucose. Injection of rats with LPS caused a significant liver dysfunction and this liver dysfunction was only moderately inhibited by treatment of endotoxemic rats with the combination of TB plus GB. Administration of rats with LPS induced a significant kidney dysfunction and this kidney dysfunction was not ameliorated by the treatment of rats with TB combined with GB. LPS administration elicited dramatic increases in plasma NO and aortic O2- levels, and TB combined with GB attenuated these increments. In particular, the aortic $\mathrm{O} 2$ - formation was almost completely abolished by these two combinations therapy. Administration of rats with LPS caused a severe mortality in mice and this is mortality was markedly reduced by the treatment of mice with TB combined with GB. 


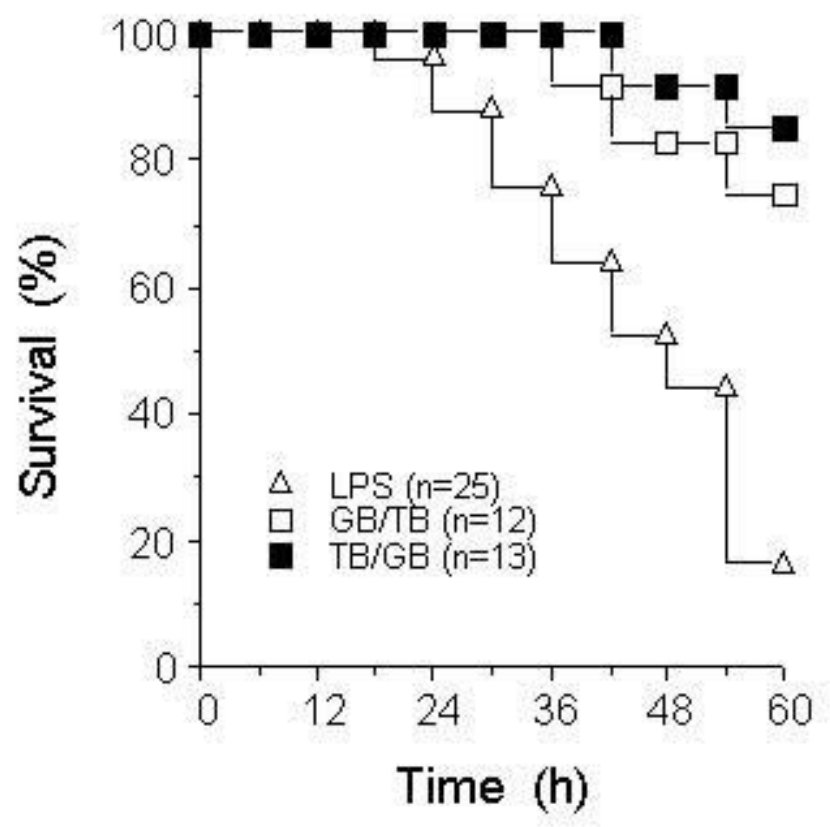

Conclusion: Our results may suggest that (1) the blockade of NO and O2-formation restore the hemodynamic changes and (2) other mediators contribute to the organ dysfunction elicited by endotoxin-associated septic shock. In addition, the administration of TB combined with GB may improve the survival of septic animals or patients 